\title{
Cellular dynamics observed at sub-nanometer resolution using atomic force microscopy
}

Tiina Lehto, Harald Janovjak, Clemens Möller, Fedor Severin, Marta Miaczynska, Marino Zerial, Galen Hand, Gina Sosinsky, Daniel J. Müller

Max-Planck-Institute of Molecular Cell Biology and Genetics, Pfotenhauer Str. 108, D-01307 Dresden, Germany

Single molecule experiments provide insight into the individuality of biological macromolecules, their unique function, their reaction pathways, their trajectories and into their molecular interactions. The exceptional signal-to-noise ratio of the atomic force microscope (AFM) allows individual proteins to be imaged under physiological relevant conditions at a lateral resolution of $0.5-1 \mathrm{~nm}$ and a vertical resolution of $0.1-0.2 \mathrm{~nm}$. This capability is reviewed on various watersoluble and on membrane proteins. Lately, it became possible to observe molecular processes at the single-molecule level using this technique. Examples observing function, variability, and assembly of single proteins are discussed. Recent developments of scanning probe microscopy techniques enable measuring simultaneously multiple biochemical signals on individual macromolecules. Recorded with submolecular resolution these signals can be directly assigned to structural details of individual proteins of a cellular membrane. Examples discussed are the detection of structural variability and flexibility, of surface charges, and of electrostatic potentials of transmembrane ion channels.

The recently invented combination of single-molecule imaging and forcespectroscopy enables the controlled manipulation of single proteins to detect their inter- and intramolecular interactions. The experiments allow observing the unfolding pathways and forces of secondary structural elements of biological macromolecules such as $\alpha$-helices, $\beta$-sheets and, most surprisingly, of polypeptide loops. In future these and forthcoming methods will provide novel molecular biological insights into factors determining structure, stability and function of individual proteins and of their assemblies.

In recent approaches we combined above techniques to gain insights into vesicle transport, fusion and signal trafficking of native cell biological systems. First results of the experiments will be presented showing dynamics of complex cellular systems at molecular resolution.

Key words: ATP synthase, cytoskeleton, connexon, gap junction, intracellular communication, porin, protein folding, signal trafficking, vesicle fusion, vesicle transport, 

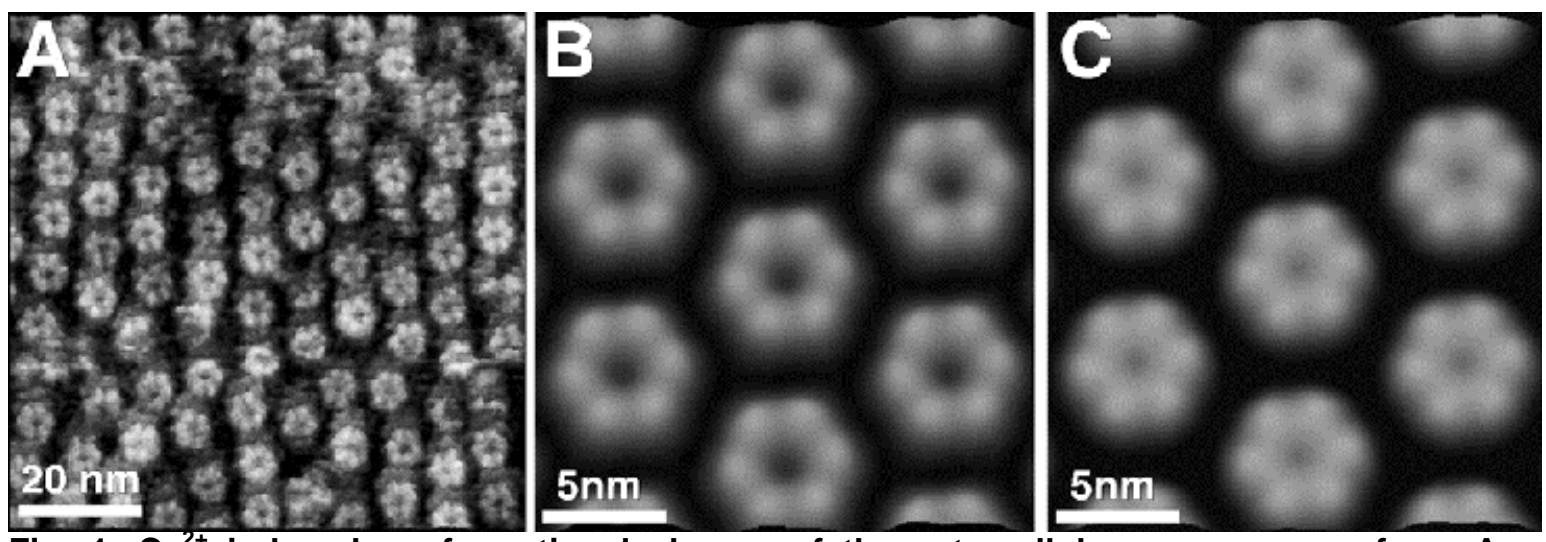

Fig. 1. $\mathrm{Ca}^{2+}$ induced conformational change of the extracellular connexon surface. $\mathrm{A}$, Extracellular connexon surface imaged in buffer solution ((5 mM Tris, $1 \mathrm{mM}$ EGTA and $1 \mathrm{mM}$ PMSF). B and C, averaged topographs of extracellular connexon surfaces recorded in absence of $(\mathbf{B})$ and with $0.5 \mathrm{mM} \mathrm{Ca}^{2+}(\mathbf{C})$. The contact mode AFM topographs were recorded using applied forces of $50 \mathrm{pN}$, a line frequency of $5.5 \mathrm{~Hz}$ and were displayed with a vertical scale of $2 \mathrm{~nm}$. (Müller et al. EMBO J. in press)

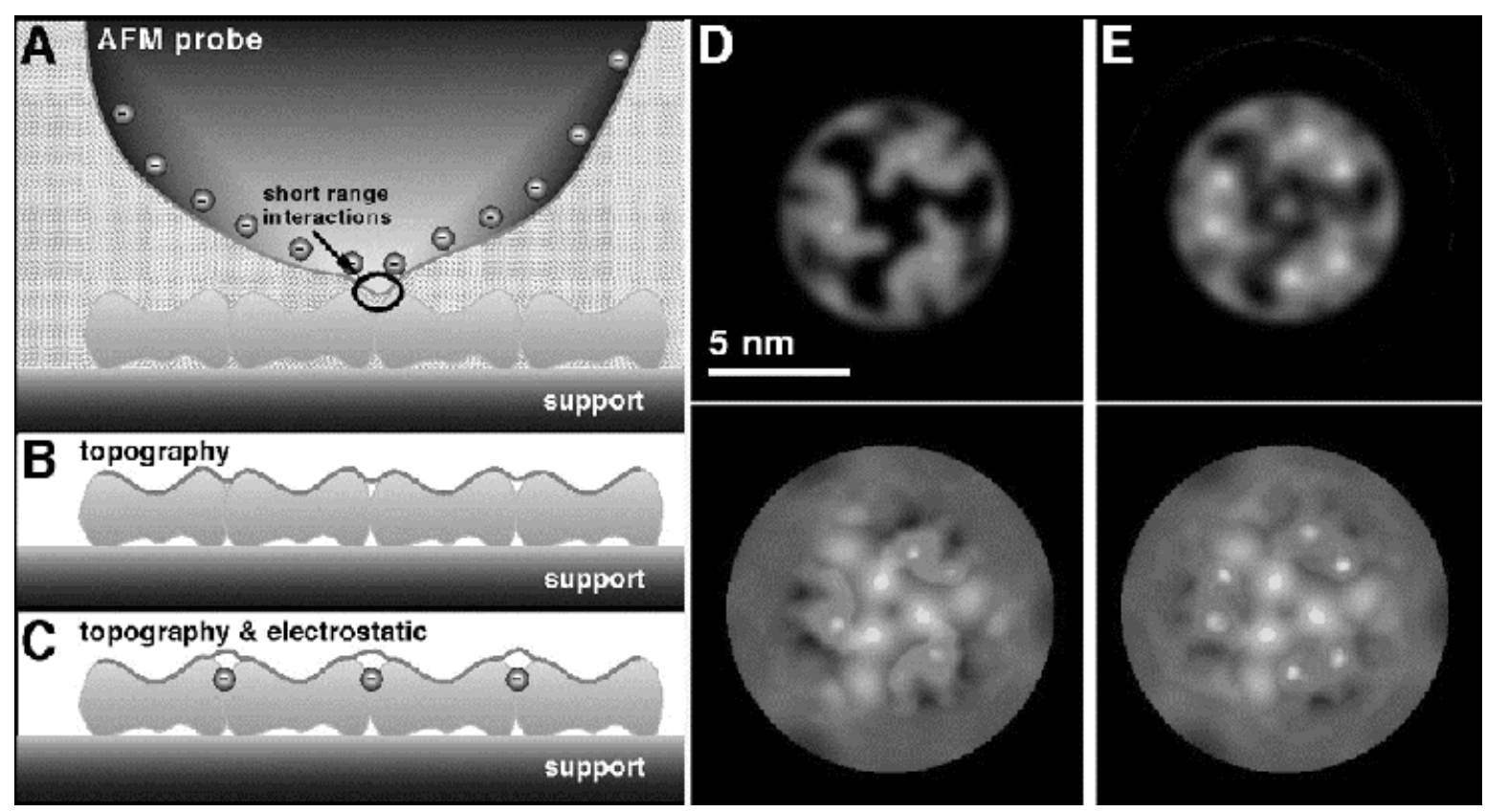

Fig. 1. Mapping the electrostatic potential of the OmpF porin channel. A, Principle of recording topographs at variable electrostatic contribution: B, Scanning a negatively charged AFM tip (silicon nitride) over an electrically neutral surface reveals the true topography. C, In case of discrete negative charges the AFM tip detects local electrostatic repulsions. The resulting topograph represents a mixture of structural and electrostatic information, which can be decomposed by subtracting the topograph recorded without from that recorded with electrostatic contributions. Difference maps between topographs recorded of the periplasmic OmpF porin surface at 100 and $300 \mathrm{mM} \mathrm{KCl}$ and at 50 (D) and $300 \mathrm{mM} \mathrm{KCl}(\mathbf{E})$. As becomes evident, the main differences between porin trimers recorded at different electrolyte concentrations are located at the entrances of the transmembrane pore. The color scale shaded from white (highest difference) to red (high difference) to black (difference $<0.05 \mathrm{~nm}$ ) corresponds to a vertical height of $0.3 \mathrm{~nm}$ (D) and of $0.5 \mathrm{~nm}(\mathrm{E})$. Lower panels show the superimposition of the averaged topograph (colored brown-gold) and the electrostatic potential. (Philippsen et al. Biophys.J. in press). 
https://doi.org/10.1017/S1431927602102339 Published online by Cambridge University Press 